\title{
L'esprit dans les récits poétiques bibliques de Jean Grosjean ${ }^{1}$
}

\author{
Aurélia Hetzel, École Pratique des Hautes Études, Université Paris IV-Sorbonne
}

«Heureux, vous qui pleurez maintenant, car vous rirez ${ }^{2} »($ Luc 6, 21).

«Malheureux, vous qui riez maintenant! car vous connaîtrez le deuil et les larmes » (Luc $6,25)$.

La Bible n'est pas un livre drôle. Il est d'autant plus tentant d'en rire, peut-être aussi particulièrement facile : entre son caractère sacré, trop figé peut-être, et sa prétention à guider la vie humaine, il existe un hiatus dans lequel peuvent prendre place toutes les formes d'humour. Certes, dans une perspective chrétienne, «la vie sur terre consiste à s'associer à ce sacrifice [du Christ] en recherchant la souffrance (source de mérites) et en fuyant le plaisir (source de péché). Une telle perspective laisse peu de place au comique » (Cerbelaud 107). Les Pères de l'Église nous rappellent sévèrement que le Christ ne rit jamais dans les Évangiles ${ }^{3}$. On peut donc être surpris que les réécritures bibliques ${ }^{4}$ de Grosjean (1912-2006) soient empreintes d'un certain sens de l'humour. Pour ce poète chrétien, prêtre jusqu'en 1950, il ne s'agit pas de se moquer de la Bible, encore moins de la décrédibiliser : à aucun moment il ne la tourne en ridicule, ni ne fait rire à ses dépens ; on peut même considérer parfois son humour comme un jeu gratuit. C'est qu'il ne s'agit pas, dans ses réécritures bibliques, de rire de la Bible, mais plutôt de rire avec la Bible, avec des personnages que des siècles de littérature ont rendu disponibles dans l'imaginaire, et que l'on peut remettre en scène dans d'autres contextes; des archétypes, selon Grosjean, qui insiste cependant sur le caractère individuel de l'expérience chrétienne, et qui confère donc à ses personnages une dimension très humaine.

Comment Grosjean nous fait-il rire, sourire, ou comment au moins nous donnet-il un plaisir humoristique? D'une part, l'hypotexte biblique de ces réécritures poétiques étant célèbre, le narrateur joue de la complicité qu'il entretient avec le lecteur, qui connaît le destin des personnages avant eux-mêmes. D'autre part, puisqu'il s'agit de récits qui se veulent à la fois traductions, exégèses et transpositions en utilisant d'autres mots, sans trahir l'esprit biblique, le lecteur n'est pas étonné de trouver autant de jeux de 
mots dans les récits de Grosjean. On se situe ici dans une perspective chrétienne, où il faut garder à l'esprit que le Verbe s'est incarné dans le Christ.

\section{Une relecture complice}

« À chaque génération la charge de retraduire l'original (Araméennes 118). Selon Grosjean, pour que la Bible soit transmissible, il faut qu'elle apparaisse comme un univers familier à ses lecteurs : elle n'est pas écrite une fois pour toutes, mais elle est à réécrire et à relire par chacun. L'humour de cet écrivain, qui mêle au substrat biblique des éléments, lieux, événements ou expressions connus du lecteur contemporain, permet à ce dernier d'accéder au texte sacré directement, en se libérant d'une lecture imposée, forcément figée, évidemment inadaptée, qui risque d'être peu fertile. Or, c'est justement l'humour soulignant ces décalages qui permet ici la réconciliation entre l'ancien et le moderne, le sacré et le profane. Grosjean ne nie pas les écarts, mais il les dépasse. Ainsi, dans Les Beaux Jours, le Christ « se mêle aux gens qui vont à l'église. Beaucoup le reconnaissent et pas mal le saluent » (21) : l'incongruité n'est qu'apparente ; la présence du Christ dans une église est évidente, puisque, corps et âme, il est l'incarnation du spirituel. L’image, des représentations iconographiques sacrées à la représentation littéraire, résout l'anachronisme.

De plus, la distance prise avec l'hypotexte confère un caractère profondément humain aux personnages bibliques. Ainsi, au livre XIII des Juges, un ange apparaît à la mère stérile de Samson pour lui annoncer qu'elle va enfanter un fils qui sera nazir, c'est-à-dire consacré à Dieu. Dans le récit de Grosjean, Samson, cet épisode, qui est l'un des éléments constitutifs de l'histoire de Samson, est condensé en une seule phrase de la mère : «c'était bien la peine qu'un ange se soit déplacé pour nous promettre cette espèce de garnement»(19). Le registre familier annule le caractère extraordinaire, comme souvent chez cet écrivain, peut-être parce que les miracles ne concernent que le Christ : Samson, dont la mère dit qu'il est «fort pour son âge » (7), est ramené à une dimension parfaitement humaine.

L'humour de Grosjean repose donc essentiellement sur le décalage: déplacements dans le temps, dans l'espace ou dans le registre, ces écarts font rire parce qu'ils surprennent le lecteur, qui les mesure par rapport au texte biblique. Or, l'un des aspects humoristiques de ces textes naît justement du décalage entre les personnages et 
leur propre histoire, déjà écrite, déjà connue, et qu'ils n'ont pourtant pas encore vécue. On a donc une feinte du narrateur, en même temps que trop de désinvolture le trahit. Cette ironie crée une complicité entre le narrateur et le lecteur, aux dépens du personnage, dont la naïveté est amusante. Par exemple, dans les versions juives et musulmanes de la légende de la reine de Saba, notamment dans le Targum Shéni d'Esther et dans le Coran (Sourate XXVII, «Les Fourmis »), Salomon fait passer une épreuve à la reine qui vient le voir à Jérusalem : il lui demande de traverser un sol dallé de cristal, sous lequel il y a de l'eau, parfois même des poissons ; la reine relève donc sa robe pour ne pas la mouiller et dévoile ainsi ses jambes au roi, qui voulait vérifier qu'elle n'avait pas des pieds de chèvre. À la suite de cela, dans le Coran, elle se convertit à l'Islam. Le motif du sol de cristal trouve sa place dans La Reine de Saba de Grosjean, mais l'épisode se conclut par une déclaration d'amour de Salomon. La scène telle qu'elle est réécrite ici est très éloignée des textes sacrés ; elle est revécue par deux êtres qui ont perdu tout caractère religieux, mais c'est pour mieux le retrouver plus tard : la reine, vexée du rire de Salomon qui la voit surprise de ne pas trouver d'eau sous ses pieds, rougit et voudrait le gifler : "Salomon a trouvé que les jambes ne déparaient du visage. Conquis et penaud il a dit : Mademoiselle, ne prenez pas pour de l'eau claire ce pavement de cristal. Vous ne le troublerez pas comme vous troublez mon cœur». La reine rit à son tour : «Elle était délivrée de jamais devoir jouer des rôles. Elle ne serait plus que l'aimée de Salomon » (32). L'épisode du sol en trompe-l'œil, qui a une haute dimension symbolique dans la mystique musulmane, apparaît ici à première lecture comme une blague de Salomon. Mais il s'agit ici, d'une part, de se conformer aux rôles qu'ont fait jouer les traditions à ces personnages. Cette scène est indispensable dans une réécriture de l'histoire de la reine de Saba, ce qui fait, peut-être, du piège de Salomon, un tour qui marche à coup sûr, dans toutes les réécritures, à tel point qu'on ne peut tout de même plus le prendre au sérieux et qu'il n'y a que la reine de Saba pour s'y laisser encore prendre. D'autre part, ce rire du roi, auquel fait presque immédiatement écho celui de la reine, permet d'humaniser les personnages et de leur donner un corps. Les procédés humoristiques tentent justement de faire se mouvoir ces personnages sacrés, figés dans les textes religieux. De même, tout en cherchant à recréer la spiritualité biblique, car la dimension chrétienne n'est jamais occultée, le narrateur met en scène la vie la plus quotidienne des personnages : 
Mes récits ne s'intéressent guère à l'histoire du monde; ils s'intéressent pourtant à l'expérience de la durée, au sens d'une durée de la vie [...] Dire ou entendre l'histoire d'une nation, d'une société quelconque, même d'une famille, m'ennuie plutôt. En réalité, on n'a jamais d'autre expérience que la durée d'une vie humaine [...] Quand le Messie est venu remplir toute l'Écriture au cours de sa seule vie humaine, il substituait à l'histoire du monde une biographie. (Araméennes 29-30)

On suit souvent l'histoire des personnages presque au jour le jour, et cette linéarité donne toute leur cohérence aux récits. Dans Élie, Jézabel fait du repassage (90) ; dans Samson, on mange «des œufs sur le plat à la lueur d'une bougie » (18). On peut lire dans ces éléments une sorte de fantaisie burlesque, mêlant profane et sacré. Pour rendre son récit contemporain en abolissant les frontières du temps et de l'espace, Grosjean fait coïncider son expérience, la nôtre et celle de ses personnages. On voit donc bien à quel point sont superposés le passé et le présent, le sacré et le profane sous le regard englobant du narrateur. L'humour permet de passer naturellement de l'un à l'autre.

Enfin, ces récits créent, dès leur titre, un horizon d'attente constitué de certains lieux communs auxquels ils ne sauraient se dérober : Jonas doit être jeté à la mer, Pilate finit par se laver les mains. Dans Adam et Ève, le couple originel se retrouve sur les bancs de l'école. Ève, avide de connaissances, est une bonne élève, tandis qu'Adam est plus réticent et finit par regretter « le charme de ne rien savoir» (103). Les motifs éparpillés par petites touches allusives fonctionnent comme des indices et créent un réseau de repères intertextuels. C'est la reconnaissance de ces éléments attendus qui fait sourire : on a plaisir à les découvrir ou à les dénicher dans le texte, on s'amuse de la plus ou moins grande subtilité avec laquelle le narrateur les a dispersés.

Mais si l'on rit ou sourit volontiers des anachronismes par rapport à la Bible, ces écarts ne sont pas toujours aussi drôles. Au contraire, ils sont parfois l'expression d'un humour grinçant, comme dans cette phrase de La Reine de Saba : «le roi [Salomon] avait royalement logé ses Sarrois au Lutétia, au Lucullus et au Lustucru avec vue sur les pittoresques fumées de la Géhenne » (48), où l'on peut lire une allusion à la Seconde Guerre Mondiale. Après avoir été le siège de l'Abwehr pendant la Seconde Guerre Mondiale, le Lutétia, hôtel parisien luxueux de la rive gauche, se transforma en centre d'accueil pour les déportés, et la Géhenne, qui désigne l'enfer dans la Bible, un enfer où l'on brûle, évoque aussi les cheminées des camps de concentration. Cela s'oppose au grand raffinement gastronomique dans lequel vivait Lucullus et à la célèbre marque de 
produits alimentaires Lustucru. Les allusions à cette guerre, tout comme à celle de 1914-1918, très nombreuses dans ses récits, évoquent une période extrêmement douloureuse de la vie du poète, né deux ans avant la première guerre et prisonnier entre 1940 et 1942. On peut aussi voir dans l'humour de Grosjean une sorte de thérapie, une «anesthésie momentanée du cœur» (Bergson 4). Interrogé sur l'humour diffus qu'il y a dans ses œuvres, il fait cette réponse, qui peut passer à première lecture pour une boutade :

Au moment où les choses sont trop fortes, il vaut mieux en rire qu'en pleurer. Et il me semble, lorsque je me mets dans la peau d'un personnage, que je le vois sous son côté souvent douloureux qui m'intéresse. Mais je pense qu'avec un certain recul, Dieu le voit plus drôlement que lui ne se voit. (L'Eil de bœuf 15)

Le rire comme purification de la douleur peut sembler aux antipodes des théories chrétiennes, notamment celles du sacrifice et de la méfiance vis-à-vis du plaisir. Or, Grosjean dépasse la contradiction, car il ne se situe pas du tout dans une perspective morale ; il s'agit pour lui de célébrer le Christ, vers lequel convergent, selon lui, toutes les grandes figures bibliques. Dans L'Ironie christique, il explique :

Jésus ne s'esclaffe pas, il ne s'est jamais esclaffé, il rit en silence. Enfant sa curiosité de Dieu le rendait grave, mais quand il s'est su le Fils, le sourire l'a gagné. Même ses colères se sont teintées d'humour: dérisoires les soucis, naïves les intentions, incroyables les fourvoiements. Même dans l'angoisse il a eu des ironies. Et maintenant après l'horreur, il rit tout bas mais franchement et sa réponse est une question. (270)

Le sérieux de ces réécritures chrétiennes, qui célèbrent le Christ de manière très poétique, et la légèreté avec laquelle le poète semble parfois traiter ses personnages forment un contraste à la fois grave et amusant. C'est justement cette équivoque qui crée une distance permettant de ressaisir le message biblique.

\section{Du langage créateur au langage créatif}

Ce sont les jeux sur le langage qui permettent le mieux d'exprimer cette équivoque $^{5}$. Chez Grosjean, il s'agit le plus souvent de calembours, «qui passent pour la variété la plus basse du mot d'esprit fondé sur des mots, vraisemblablement parce qu'ils permettent de faire de l'esprit 'à bon marché' et en se donnant le moins de peine possible $^{6} \gg$ (Freud 104). Ils peuvent parfois effectivement sembler gratuits, comme celui-ci dans La Reine de Saba: «Elle n'éprouvait guère le besoin qu'ils aillent la 
décrire à quelque pharaon incestueux ou à quelque chah cynophile » (30), mais peut-être faut-il lire ici une allusion à l'animalité controversée de la reine ou, plus prosaïquement, une anticipation sur ses rapports chien et chat avec le roi. De même, dans Élie, on peut lire :

Le roi s'approcha d'Élie en humble complice. Élie ne le repoussait pas. Sans le regarder il semblait l'accepter. Les gens se disaient : Qui trompent-ils ces deux-là ? Il faudra que quelqu'un paie. On a été abusé par quelqu'un. On va se retrouver grosjean comme devant (44).

Difficile de lire ici plus qu'un clin d'œil gratuit, mais le jeu de mot, si facile soit-il et qui n'a pas non plus pour ambition de faire s'esclaffer le lecteur, le fait quand même au moins sourire et contribue ainsi au plaisir savoureux que l'on éprouve à la lecture de ces récits poétiques.

Grosjean s'amuse aussi beaucoup avec les expressions où l'on trouve le mot Dieu. Par exemple, lorsque Samson tue un lionceau, la Bible dit : «L'esprit de Yahvé fondit sur lui » (Juges 14, 6) ce qui donne dans le texte de Grosjean : «Dieu merci, je l'ai tué » (Samson 31). La locution adverbiale «Dieu merci » est figée au point que la mention de Dieu a perdu tout son caractère sacré ; l'humour naît de la pseudo naïveté de cette formule. De même, dans Élie, Obadyahu, le maître du palais d'Achab et protecteur des prophètes, dit à Élie qu'il rencontre par hasard alors que le roi le fait chercher : «tu seras Dieu sait où » (36) («l'Esprit de Yahvé t'emportera je ne sais où »1, Rois 18, 12). Ou encore dans Jonas, lorsque le bateau sur lequel s'est embarqué Jonas est pris dans la tempête : «Les gars, cria le capitaine, il faut tirer à la courte paille. On saura qui nous vaut ce ramdam. Ce n'est pas Dieu possible qu'il n'y ait pas sur le bateau quelqu'un contre qui le ciel se déchaîne » (35-36). Selon Sigmund Freud : «le mot d'esprit réalise justement les conditions dans lesquelles des mots dont le sens primitif a pâli récupèrent leur plein sens » (90). Ici, l'écart entre les expressions avec «Dieu » et leur sens plein est effectivement comblé, en même temps qu'il permet de re-sacraliser l'expression profane.

Mais les calembours peuvent aussi inviter à une réflexion sur le langage. Ainsi, dans Pilate : «Barabbas ou Jésus, le choix aurait pu être embarrassant, on aurait pu voir l'ambivalence des mots, car Barabbas s'appelait lui-même Jésus et Jésus était lui aussi Fils du Père» (55). Certains manuscrits bibliques appellent Barabbas «JésusBarabbas », signifiant en araméen « fils du Père », ce qui est justement le titre de Jésus. 
Le jeu de mots ne rappelle rien de plus, et finalement suggérer une équivalence entre Barabbas et «embarrassant» ne fait qu'insister sur l'embarras de Pilate. En même temps, il permet ici de renforcer l'identification entre Barabbas et Jésus face au peuple : «Mais les guides des foules n'y regardent pas de si près. Comme que se nomme qui que ce soit, on s'en moque, on veut la peau de celui qui fait de l'ombre » (55). En jouant sur les mots, le poète rappelle donc, avec cette simplicité déconcertante qui lui est propre, l'importance des mots et des noms : après tout, «D'abord il y avait le langage et le langage était chez Dieu, et le langage était Dieu. Il était d'abord chez Dieu. C'est par lui que tout a existé et rien de ce qui existe n'a existé sans lui. C'est en lui qu'était la vie et la vie était la lumière des hommes » (Évangile de Jean, I, 1, traduction de Grosjean 11). Et Jésus est celui qui n'a pas été reconnu.

Enfin, de calembours en jeux de mots, on assiste parfois à une véritable fête du langage, comme au chapitre final de Samson. Ainsi, la présentation du vieux Bia, organiste, est amenée par le jeu de mots entre «les tuyaux [des courses] »et «l'orgue » (93). Ce nom fait l'objet de jeux de mots : les deux premiers, «art à Bia » et «char à Bia » (93), présentent, pour l'oreille, deux sens. Le complément du nom est introduit par la préposition «à » au lieu de « de », ce que l'on trouve précédemment dans «la fête à Dagon » (92), créant une ambiguïté entre nom de lieu et nom de Dieu. On trouve plus loin «le petit Bia», jeu de mots renforcé par «qu'on appelait Péroné » (93). Ce petit Bia rit de Samson, que le meunier a fait se «hâter » (93), vocable que l'on peut rapprocher de «bâter », Samson étant assimilé à un âne. Or, ceux qui devaient rire font rire, en jouant une espèce de farce carnavalesque : l'idée introduite par «charabia » (quelques chapitres auparavant, Samson avait déjà été placé sur un « char à bœufs ») est reprise avec le terme «charivari », avec la référence à la tour de Babel, ainsi qu'avec l'incompréhension concernant Dalila : «Il faut dire qu'une légende était née à propos de Dalila libératrice. Le dieu Dagon lui aurait dépêché l'ange du Nois quand elle était à glaner du bois mort (d'autres disaient : quand elle gardait les deux chèvres de son oncle Niort)»(96). De plus, sont présents à la fête des chevaux normands, des tableaux italiens, des Russes, des Bédouins, des Vendéens, ou encore des Grecs. L'écroulement du temple fait bien sûr écho à celui de l'entreprise de la tour de Babel et ce parallélisme ne peut que s'exprimer par une fête du langage, un jaillissement de jeux de mots et de néologismes, qui s'achève par le seul bruit des «pas du jardinier galiléen dans des 
frémissements d'herbe » (98), que Samson est seul à entendre et qui appartiennent à un autre monde, à un autre rassemblement, celui de ceux qui sauraient entendre et voir. Le rire provoqué par la «fête à Dagon » permet d'anéantir les païens, de s'en exorciser, et cette purification prépare à la rencontre avec le Christ, inévitable pour Samson le sacrifié, annoncé au début du chapitre comme «le clou de la fête» (93). Le rire, manifestation physique de la joie, ou du moins d'un certain plaisir mental et spirituel, n'est donc peut-être pas toujours blasphématoire. Au contraire, il semble que l'humour de Grosjean transforme la lecture biblique en véritable plaisir, donnant accès à des correspondances dans le Livre même, et entre celui-ci et le monde profane dans lequel il est lu.

On a souvent envie de donner leurs lettres de noblesses aux traits humoristiques que l'on relève dans une œuvre et de les justifier au nom de la profondeur et de la gravité que l'on attend de la part d'un grand écrivain : le trait d'humour est censé avoir un but plus élevé que le simple sourire, il est censé expliquer, révéler, condamner, faire réfléchir ; l'humour gratuit est dédaigné, peut-être tout simplement parce qu'il est moins drôle, mais peut-être aussi parce qu'on attend plus de sérieux d'un écrivain chrétien comme Grosjean ? Si son humour permet de révéler, ou de réveiller, certaines idées, ou d'opérer certains rapprochements, il peut d'autres fois sembler gratuit, et bien sûr il n'est pas indispensable au message biblique. Or, ses réécritures bibliques, qui laissent une large place à la fiction et à la poésie, sont surtout des oeuvres littéraires. Et l'humour est aussi une part du plaisir littéraire, d'autant plus qu'il libère ici les mots de la Bible de leur carcan sacro-saint.

\section{Bibliographie}

Bergson, Henri. Le Rire. Essai sur la signification du comique. Paris: Quadrige / P.U.F., 1991 [1900].

\section{Bible de Jérusalem.}

Cerbelaud, Dominique. «Figures de l'humour chrétien. De l'irrévérence humaniste à la folie en Christ ». Humour et religions. Humoresques 12 (juin 2000).

Doublet, Élisabeth. L'Autre côté du langage : l'humour et l'ironie dans les poèmes et récits de Jean Grosjean. Besançon : PU Franc-Comtoises, 2002.

Grosjean, Jean. Le Messie. Paris : Gallimard, « N.R.F. », 1974. 
---. Élie. Paris : Gallimard, « N.R.F. », 1982.

---. Darius. Paris : Gallimard, « N.R.F. », 1983.

---. Pilate. Paris: Gallimard, « N.R.F. », 1983.

---. Jonas. Paris : Gallimard, « N.R.F. », 1985.

---. La Reine de Saba. Paris : Gallimard, « N.R.F. », 1987.

---. Araméennes. Conversations avec Roland Bouhéret, Dominique Bourg et Olivier

Mongin. Paris : Cerf, « Parole présente », 1988.

---. L'Évangile selon Jean. Nouvelle traduction de Jean Grosjean. Paris : Gallimard, « N.R.F. », 1988.

---. Samson. Paris : Gallimard, « N.R.F. », 1988.

---. L'Ironie christique. Commentaire de l'Évangile selon Jean. Paris : Gallimard, «N.R.F. », 1991.

---. Revue L'Eil-de-bøuf, n²1, juin 1993.

---. Samuel. Paris : Gallimard, « N.R.F. », 1994.

---. Adam et Ève. Paris : Gallimard, « N.R.F. », 1997.

Freud, Sigmund. Le Mot d'esprit et sa relation à l'inconscient. Tr. de l'allemand par Deis Messier. Paris : Gallimard, «Folio Essais », 1988 [Der Witz und seine Beziehung zum Unbewussten. Leipzig et Vienne, 1905].

Lanson, Gustave. L'Art de la prose. Paris : Fayard, 1908.

Mayaux, Catherine (éd.). Jean Grosjean poète et prosateur. Actes du colloque de Besançon (janvier 1997). Paris : L’Harmattan, « Publication du centre JacquesPetit », 1999.

Roudaut, Jacques. «De l'ellipse à l'énigme ». Paris : «N.R.F. », n 479, décembre 1992.

\section{Notes}

${ }^{1}$ Cet article est la version remaniée d'une communication lors du $7^{\mathrm{e}}$ colloque international de CoRHum : Faire rire : mode d'emploi, Nanterre, France, juin 2007.

${ }^{2}$ Sauf précision, les citations bibliques sont extraites de la Bible de Jérusalem.

${ }^{3}$ Mais, selon Grosjean, il y fait preuve d' « une singulière ironie qui se mêle à la tendresse comme à l'hostilité, à l'étonnement comme à la souffrance. Jésus aime laisser entendre un peu autre chose que ce qu'il dit et il y emploie l'oblicité avec tant de droiture que l'interlocuteur en est pantois ou embarrassé [...] Il semble que Jésus ait remplacé la dimension du sacré que le judaïsme avait emprunté aux païens, par l'ironie qui ne vise que l'amour-propre » (L'ironie christique 28).

${ }^{4}$ Nous nous concentrerons, dans le cadre de cet article, sur les textes suivants : Le Messie (1974), Les Beaux Jours (1980), Élie (1982), Darius (1983), Pilate (1983), Jonas (1985), La Reine de Saba (1987), Samson (1988), Samuel (1994) et Adam et Ève (1997), publiés chez Gallimard, dans une collection qui leur est entièrement consacrée. 


\footnotetext{
${ }^{5}$ Voir Jacques Roudaut : «L'ironie que Jean Grosjean dit 'christique', c'est-à-dire liée à la parole, ne consiste pas en une forme d'antiphrase ; elle ne fait pas entendre le contraire de ce qu'elle dit : elle fait triompher l'équivoque » (148).

${ }^{6}$ De même pour Gustave Lanson : «le calembour est la forme la plus basse du sentiment des sonorités verbales : voilà pourquoi il lui arrive de rapprocher les grands artistes des grands imbéciles » (32).
} 